\title{
DESARROLLO TERRITORIAL BAJO SEQUÍA Y CENIZAS
}

\author{
Ana María Murgida ${ }^{1}$ \\ Fernando Martín Laham ${ }^{2}$ \\ Carlos Juan Pedro Chiappe $\mathbf{3}^{3}$ \\ Martín Ariel Kazimierski ${ }^{4}$
}

\section{Introducción 5}

Nuestro estudio de caso se encuentra en el área rural de influencia de la localidad de Ingeniero Jacobacci, que incluye los Departamentos 25 de Mayo y Ñorquinco de la Región conocida como la Línea Sur de la provincia de Río Negro en la Patagonia argentina (ver Mapa 1). Dicha población se caracteriza por la convivencia de culturas en una estepa con un clima árido, donde la forma de producción por excelencia es la ganadería ovina.

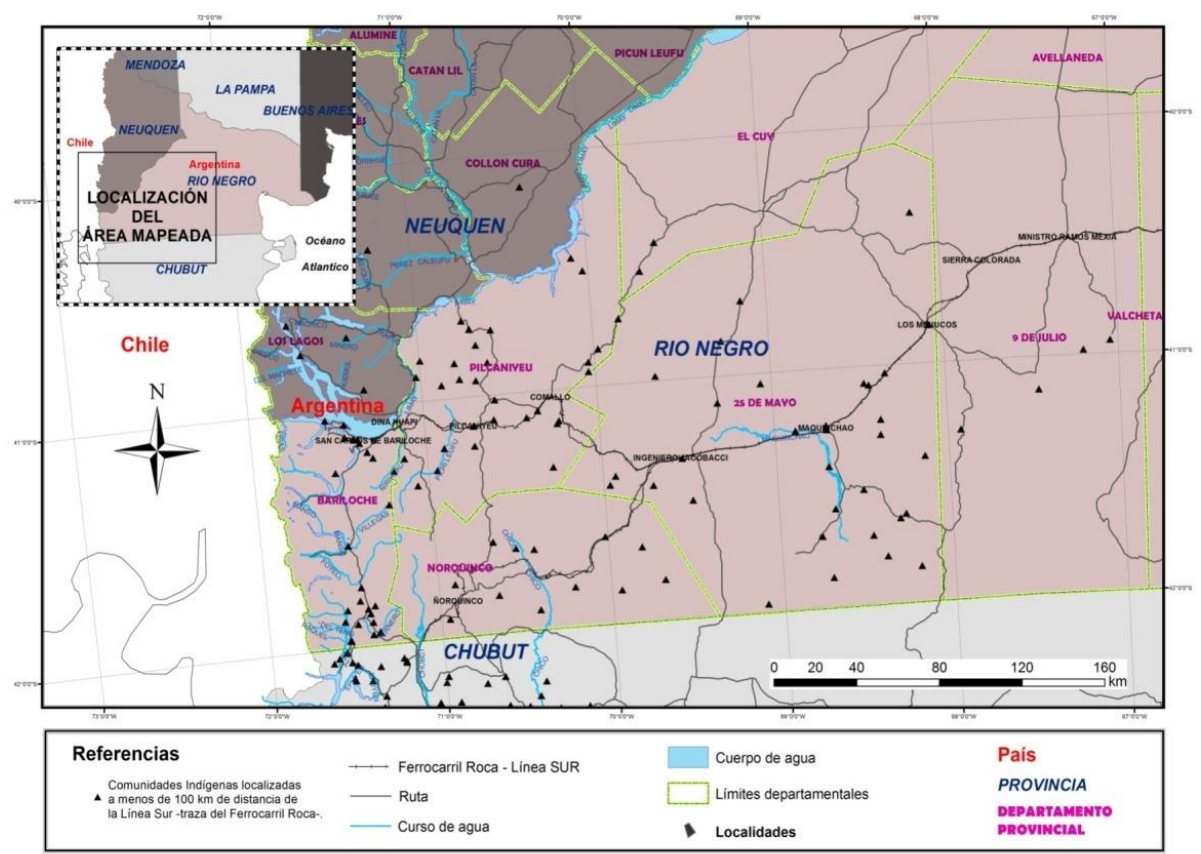

Mapa 1: Ubicación del área de estudio en la Línea Sur. Fuente: elaboración propia.

\footnotetext{
${ }^{1}$ Universidad de Buenos Aires, Argentina.

${ }^{2}$ Universidad de Buenos Aires, Argentina.

${ }^{3}$ Universidad Atlántida Argentina y Universidad Nacional de Tres de Febrero, Argentina.

${ }^{4}$ Universidad de Buenos Aires, Argentina.

${ }^{5}$ Este trabajo se realizó con la subvención del Instituto Interamericano para la Investigación del Cambio Global (IAI) CRN 3102 apoyado por la Fundación Nacional de Ciencia de Estados Unidos (Grant GEO1128040); del International Development Research Centre (IDRC) Grant 107097 y del UBACyT Interdisciplinario 2013-2016.
}

Iluminuras, Porto Alegre, v. 17, n. 41, p.11-29, jan/jun, 2016. 
La estepa está signada por transformaciones históricas relativas a la distribución del territorio y de los riesgos socioambientales. Los riesgos se caracterizan por una serie de restricciones socioeconómicas, fundiarias, de acceso a la tierra, al agua así como a los mercados, bajo el marco de una fuerte degradación de los recursos, y de situaciones catastróficas tales como sequías, nevadas y desertificación.

Eventos recientes como la sequía que se inició en 2006, y se prolongó con rudeza hasta 2014. La caída de cenizas por la erupción en los complejos volcánicos el Chaitén en mayo de 2008, y el Cordón Puyehue-Caulle en junio de 2011, potenciaron sus efectos, dando lugar a una situación catastrófica que requirió de la atención técnica y financiera de instituciones gubernamentales y atrajo la atención de organismos no gubernamentales y científicos.

Esta convergencia entre profesionales de distintas formaciones, procedencias y praxis, en esta área donde la población sufre un proceso catastrófico de larga duración, dio lugar a una interconsulta que decidimos transformar en un ejercicio de reflexión, que respete la contribución colectiva al conocimiento, con resultados documentados, entre ellos, este artículo.

La población de la estepa, en su mayoría mapuche, está organizada en comunidades que a su vez constituyen el sujeto de derecho del Instituto Nacional de Asuntos Indígenas (dependiente del Ministerio de Desarrollo Social de la Nación) y, en el marco del proyecto de investigación, buscamos comprender los modos adaptativos bajo condiciones hidroclimáticas extremas. La sinergia de nuestra relación busca generar lineamientos integrales de abordaje territorial que contribuyan a las tomas de decisión. Especialmente analizamos la potencialidad de incluir la vulnerabilidad y la adaptación sociales frente a las amenazas múltiples a la hora del diseño de políticas gubernamentales.

El acuerdo conceptual de base es que el riesgo es una construcción definida por las condiciones sociales, económicas, culturales e institucionales, que incluye la interacción en un tiempo y territorio específicos con eventos físico-naturales (Douglas, 1996; García Acosta, 2005; Lavell, 2005). Consideramos relevante analizar la construcción del riesgo en la relación dinámica existente entre las amenazas, las vulnerabilidades sociales y las formas de adaptación. 
El riesgo y la catástrofe son procesos que se construyen y se reconstruyen con el paso del tiempo. Es decir, además de los fenómenos de origen natural existe una acumulación histórica de vulnerabilidades y amenazas derivadas de las intervenciones territoriales, que inciden en la intensidad de los efectos provocados. Su aumento y acumulación en el tiempo puede explicarse por la incidencia de eventos físicos construidos o amplificados socialmente, lo que incluye las formas de organización social y las decisiones gubernamentales (García Acosta, 2002; Lavell, 2005; Murgida et al, 2014).

En este proceso, tiene un peso considerable la adaptación, es decir, en el modo de desarrollo bajo el cual las sociedades producen y se reproducen de manera constante. Para comprenderlo recuperamos el planteo de un antropólogo latinoamericano, Darcy Ribeiro, quien postula que el modelo de desarrollo incluye tres componentes o sistemas: el adaptativo, el asociativo y el ideológico. A partir de ello el sistema adaptativo refiere al conjunto de las prácticas de las sociedades para actuar sobre la naturaleza, proveer a su subsistencia y reproducir el conjunto de bienes y equipamiento de que dispone. Tiene como contenido especial la tecnología. Mientras que, el asociativo es el complejo de normas e instituciones que permiten organizar la vida social y regular las relaciones establecidas entre hombres y con la naturaleza así como regir la vida política. En tanto define al sistema ideológico, como el conjunto de los cuerpos de saber, de creencias y de valores generados en el esfuerzo y conductas adaptativas - asociativas (Ribeiro, 1971). Esta manera de definir la adaptación, nos permite incorporar de manera comprensiva el modo de desarrollo de la sociedad y el riesgo derivado de las formas de conducirlo desde la gestión gubernamental, y desde las prácticas de los actores sociales territoriales.

\section{El caso en la Línea Sur}

La zona árida de la Patagonia presenta factores sociales como la pobreza, la tenencia precaria de la tierra y la dispersión poblacional, así como factores ambientales tales como sequías y actividad volcánica que convergen en eventos catastróficos que

profundizan la vulnerabilidad sociocultural. Ésta deriva a su vez de la disputa histórica por la ocupación de la tierra y la consideración o no del riesgo en el diseño de las políticas públicas (Murgida \& Gentile, 2015). Desde el punto de vista de una definición productiva, 
la estepa patagónica se incorpora como territorio de ganadería ovina inscripto en un sistema lanero. Para la acción social, ésta quedaría inscrita dentro de los sistemas productivos/reproductivos y de subsistencia tanto criollos como indígenas.

Cierto es que los productores rurales de la estepa desarrollan sus actividades en suelos de baja fertilidad bajo un clima árido, con períodos de escasez de agua para satisfacer las necesidades humanas y productivas. A la fragilidad propia del ecosistema árido se agrega la sobrecarga animal que derivó en procesos de degradación y desertificación, que incrementan la vulnerabilidad de los productores, especialmente los de subsistencia. Una característica adaptativa de la población mapuche y criolla es la organización familiar del trabajo, con unidades agropecuarias de producción y consumo con inserción en el mercado lanero internacional (LADA, 2011; Murgida \& Gentile, 2015.

Entre las formas de asociación de los productores de la estepa se destacan las comunidades y las cooperativas. Las primeras, como grupo de pertenencia de las poblaciones mapuches, que producto de procesos de redistribución y planificación del territorio desde fines del siglo XIX transformaron radicalmente las prácticas de subsistencia cazadoras-recolectoras en peonaje rural especializado en ganadería extensiva. Las comunidades originarias se definen en la Ley Nacional $N^{\circ} 26.160$ sancionada en el 2006 como las que mantienen una "ocupación actual, tradicional y pública" que se expresa sobre un territorioy que mantienen un patrón cultural y una lengua común. En la actualidad por resolución $N^{\circ} 781 / 1995$ de la Secretaría de Desarrollo Social de la Nación, las comunidades de los pueblos originarios también poseen personería jurídica como instancia de legitimación y marco "legal" ante el Estado.

La organización en comunidades constituye, históricamente, un espacio de comunalización (Brow, 1990). Esto implica la construcción estratégica de un espacio difuso que da cuenta de las tensiones tanto internas como externas de la comunidad, sin referencia necesaria a una unidad territorial jurídico-administrativa. 
La cooperativa, es una "asociación autónoma de personas que se han unido voluntariamente para hacer frente a sus necesidades y aspiraciones económicas, sociales y culturales comunes por medio de una empresa de propiedad conjunta y democráticamente controladas" (ACI, 1995). ${ }^{6}$ Las cooperativas, sujetos colectivos con personería jurídica, presentes en la zona desde los primeros años de la década del setenta, resultaron organizaciones para actuar de manera recíproca entre sus miembros y comerciar su producción lanera. En la Línea Sur, la experiencia para adaptarse al medio socio-ambiental está marcado por formas de asociación que se pueden entender como estrategias marco, que les permite contar con dispositivos sociales a la hora de enfrentar los efectos de los desastres, crisis y del mercado.

En la Línea Sur se distinguen tres tipos de cooperativas a partir de su función, sus vínculos con y entre productores individuales y otras cooperativas, sus vínculos con organismos de gobierno, organismos científico-técnicos, entes financiadores y redes de comercialización. Identificamos tres tipos de cooperativas: servicios públicos; técnica profesional; producción y comercialización, y cooperativas de segundo orden que reúne distintas cooperativas. En principio, las funciones básicas identificadas que cumplirían las cooperativas son: mediación, asociación y jurídico-administrativa. Actualmente, estas tres funciones también son asumidas por las comunidades indígenas.

Para la articulación de comunidades y cooperativas surgen organizaciones de segundo grado, en las que aquellas se nuclean y funcionan como medios de acceso a la atención pública de los problemas de estrés hídrico ${ }^{7}$ en toda la región de la Línea Sur. La asociación en entidades que van más allá de las comunidades permite incluir sectores que no se reconocen como indígenas, pero que aún así se ven expuestos y están atravesados por la misma situación.

\footnotetext{
${ }^{6}$ Alianza Cooperativa Internacional (ACI), en su Declaración sobre Identidad y Principios Cooperativos, adoptados en Manchester en 1995.

${ }^{7}$ Hay estrés hídrico cuando la demanda de agua es más importante que la cantidad disponible durante un periodo determinado o cuando su uso se ve restringido por su baja calidad. El estrés hídrico provoca un deterioro de los recursos de agua dulce en términos de cantidad (acuíferos sobreexplotados, ríos secos, etc.) y de calidad (eutrofización, contaminación de la materia orgánica, intrusión salina, etc). (UNEP, 2004).
} 
El carácter territorial, disperso y difuso de la población de la estepa constituye una morfología social compleja que no permite discriminar a aquellos integrantes de comunidades mapuches de quienes no lo son. Ambos tipos comparten las características demográficas, socioeconómicas y las prácticas productivas en el sistema bajo riesgo.

Así en esta región, la asociación en cooperativas y comunidades converge estratégicamente en un "bloque de organizaciones" que desdibuja la pertenencia o no a una comunidad indígena a la hora, en este caso, de relacionarse con programas gubernamentales.

La hipótesis que motiva este trabajo es que las diferencias en las capacidades de las poblaciones para hacer frente a situaciones socioambientales de riesgo, condicionan las respuestas adaptativas de las comunidades y de las agencias gubernamentales. Las comunidades, porque generan estrategias para acceso a los recursos naturales y tecnológicos, y las instituciones gubernamentales porque definen sus misiones en función de coyunturas sociopolíticas y perspectivas de gestión, así como de la demanda emergente del territorio. Pero especialmente los contextos de crisis y catástrofe son los que ofrecen mayores intervenciones territoriales.

Bajo este supuesto, nos propusimos desarrollar un ejercicio de transdisciplinariedad con la intención de contribuir a la gestión. Trabajamos de manera colaborativa entre profesionales y científicos de las disciplinas Antropología y Geografía, provenientes del ámbito académico, la gestión pública y ONGs sobre el caso de la población rural mapuche y criolla que habita en una región árida de la Patagonia argentina con dificultades de acceso al agua y de producción. Por esto vamos a centrarnos en la relación entre los proyectos de una institución estatal que interviene en las comunidades rurales especialmente aborígenes, con el análisis de indicadores de vulnerabilidad social. En definitiva recuperamos la vulnerabilidad social en el contexto de la adaptación social, para comprender y repensar las soluciones sustentables en función del desarrollo de la población de la región árida. 


\section{Metodología}

Esta problemática la abordamos con una doble mirada, con un trabajo colaborativo y reflexivo en el marco de un proceso de investigación que nos involucra desde diferentes roles sociales, para comprender la vulnerabilidad y a la adaptación frente a problemas ambientales y sociales, y de qué manera contribuye la gestión a la reducción del riesgo (Gibbons et al. 1994, Roux et al. 2006, Tábara and Chabay, 2013, Schmale et al, 2013).

La vulnerabilidad social es reconstruida a partir de indicadores cuali-cuantitativos. En este trabajo se consideran algunos indicadores por departamento provincial que aluden a aspectos demográficos y de composición poblacional, correspondientes al Censo Nacional de Población y Hogares del año 2010 y la variación intercensal 2001-2010, e indicadores cualitativos derivados del procesamiento de entrevistas etnográficas con la población, funcionarios y técnicos. Estos refieren a la vulnerabilidad percibida asociado a problemas de acceso y abastecimiento hídrico, como así también, programas gubernamentales y comunitarios de los que son beneficiarios. Otra fuente de datos la constituyen la bibliografía, artículos científicos, medios de prensa, así como también informes de gestión gubernamental para el período 2005 a 2014.

Para lograr el análisis complejo tomamos en cuenta las variables mencionadas, e hicimos una clasificación de los proyectos desarrollados por la institución gubernamental para establecer su relación con los aspectos cualitativos de la vulnerabilidad y las formas de asociación y adaptación en el medio sometido al stress hídrico.

\section{Resultados}

La región donde trabajamos es la Línea Sur de la provincia de Río Negro, en la República Argentina, y el caso de estudio seleccionado es el área de influencia de la localidad de Ingeniero Jacobacci. El recorte temporal se sitúa entre los años 2005 - 2014 , cuando el problema socioambiental se resume en la sequía y las cenizas de la erupción volcánica del cordón Caulle-Puyehue. El año 2006 es significativo en cuanto a la definición

entre habitantes rurales y comunidades originarias, ya que como hemos visto, la Ley Nacional $\mathrm{N}^{\circ} 26.160$ nos plantea esto y constituye a estas últimas como sujetos de derecho, introduciéndolas dentro del marco legal vigente. El año 2005 es importante pues la sequía comenzó a afectar la vida cotidiana de los habitantes rurales así como a su producción. El 
año 2008, lo es por la erupción del volcán Copahue, mientras que el fenómeno de la caída de cenizas del 2011, por la acción del Puyehue-Caulle, configuró un espacio de catástrofe al amplificar el efecto de la sequía sobre la población, su forma de producir y de circular por el territorio.

La referencia censal que empleamos corresponde al año 2010, la consideramos un proxi que leído a la luz de los resultados de análisis de vulnerabilidad social con el censo 2001 permite identificar los cambios producidos en la región y las implicancias que tuvo y tiene la gestión pública.

El indicador de población total y variación intercensal absoluta y relativa por departamento para los años 2001 y 2010 reflejan un aumento del 15\% de la población para toda la provincia entre esos años. Para los departamentos de 25 de Mayo y Ñorquinco se observa un crecimiento del casi $20 \%$ para el primero, mientras que el segundo presenta una leve disminución (ver Tabla 1). La proporción entre la población rural y urbana se refleja marcadamente para el departamento de 25 de Mayo, donde lo urbano representa casi el $80 \%$ de la población total, mientras que para Norquinco es nula, donde, a su vez, el descenso de la población rural de este último se explica por el aumento de la urbana en 25 de Mayo.

A partir de las entrevistas, se reitera la referencia de que los adultos mayores suelen permanecer en el campo haciéndose cargo de las tareas ganaderas, mientras las madres jóvenes junto a sus niños en edad escolar se trasladan al área urbana de Ing. Jacobacci (Dpto. 25 de Mayo) para su formación escolar. Esta información censal y etnográfica (trabajo de campo científico y de gestión), nos permite explicar parcialmente el desplazamiento rural-urbano dentro del área, en relación con la ocurrencia de eventos extremos que impactaron sobre el sistema productivo, pero también indica un cambio organizacional, con la incorporación cada vez más generalizada de la doble residencia de las unidades domésticas. ${ }^{8}$

\footnotetext{
${ }^{8}$ Las viviendas pertenecen al Programa Federal Reconvertido que ejecuta el IPPV junto al Municipio, y demandaron un financiamiento mixto entre Nación y Provincia de $\$ 8.046 .881$.
} 
Tabla №1: Población total y rural en Río Negro y por departamentos

\begin{tabular}{|c|c|c|c|c|c|c|}
\hline & \multicolumn{2}{|c|}{ Pob total } & \multicolumn{2}{c|}{ Pob rural agrupada } & \multicolumn{2}{c|}{ Pob rural dispersa } \\
\cline { 2 - 7 } & 2001 & 2010 & 2001 & 2010 & 2001 & 2010 \\
\hline Río Negro (prov) & 573394 & 638645 & 36.221 & 50.062 & 40.436 & 42.239 \\
\hline 25 de Mayo & 13.153 & 15.743 & 738 & 1.828 & 541 & 1.260 \\
\hline Ñorquinco & 2.079 & 1.736 & 1.017 & 1.062 & 1.225 & 511 \\
\hline
\end{tabular}

Fuente: elaboración propia en base a datos INDEC 2001 y 2010

Estos datos se logran comprender de manera más acabada cuando se observa la variación intercensal de la población rural para los censos 2001 y 2010. A nivel provincial se presenta un descenso de casi el 5\% de población rural, mientras que para los departamentos de 25 de Mayo y Ñorquinco la disminución de población rural absoluta asciende al 30\% y 16\% respectivamente (INDEC, 2001 y 2010). Estos datos reflejan un descenso de la actividad rural (LADA, 2011) en las zonas de estudio que, sobre todo en el caso de 25 de Mayo, se ve compensado por un importante aumento de la población urbana a consecuencia de los fenómenos de migración rural-urbano que afecta a la región. La variación intercensal 2001-2010 de la población rural agrupada y dispersa ${ }^{9}$ muestra una tendencia a la concentración de productores rurales en "comunidades", contra la tradicional condición dispersa de los productores rurales.

Los indicadores estructurales de vulnerabilidad social poseen un valor de aproximación y referencia de las características de la población, ya que la toma de datos censal refiere a un año particular. El valor indicativo permite el mapeo de la vulnerabilidad en el territorio y, para esta oportunidad, decidimos seleccionar algunos indicadores que podrían reflejar una articulación con los programas gubernamentales que se aplicaron en el período considerado.

Los proyectos del Instituto Nacional de Asuntos Indígenas, ejecutados y aún en desarrollo, atienden múltiples necesidades y demandas de los pobladores. Al analizarlos en sus objetivos y financiamiento, encontramos que sobre un $100 \%$ del financiamiento, se destinan directa o indirectamente en agua: 8,6\% para acceso y extracción de agua, $6 \%$ de forma indirecta para energía eléctrica y desarrollo, 7,4 \% para mejoramientos

\footnotetext{
${ }^{9}$ De acuerdo con el Instituto Nacional de Estadísticas y Censos, la población urbana es aquella que habita en localidades de más de 2000 habitantes; la población rural agrupada es la que habita en localidades de menos de 2000 y la población rural dispersa es la que habita en "campo abierto".
} 
habitacionales, 4,9\% mejoramiento productivo, $16 \%$ productivos, $1 \%$ para asistencia directa. Otros proyectos: 7,4 \% para Educación y Cultura, $32 \%$ para fortalecimiento, $12,31 \%$ relevamiento territorial y desarrollo, y 3,7\% servicios financieros.

Al revisar estas prioridades en los objetivos de los programas más representativos con los datos censales, encontramos que el Índice de Necesidades Insatisfechas (NBI), cuando es desagregado, incluye muchas de las demandas comunitarias que son atendidas por la institución gubernamental. Este índice - y sus indicadores - poseen una estabilidad relativa entre censos.

Los habitantes reconocen que las mayores amenazas son la sequía y las cenizas volcánicas. También refieren a su vulnerabilidad a través de identificar sus necesidades históricas: tenencia de la tierra, acceso al agua para consumo humano y producción, infraestructura predial y de la vivienda. También refieren algunas de las prácticas adaptativas cuando destacan la perforación de pozos para extracción de agua y la adquisición de equipamientos como bombas y tanques de almacenamiento. Asimismo el acceso a planes para el repoblamiento del ganado o el mejoramiento de infraestructura predial es reconocido en reiteradas oportunidades.

El NBI posee un valor alto para la población de la estepa, y sus indicadores se relacionan con las intervenciones del organismo de gobierno, y con las necesidades vinculadas a los efectos del estréshidroclimático y de las cenizas. Las necesidades se manifiestan en el espacio político como demandas comunitarias ante la institución gubernamental. El concepto demanda es empleado aquí en referencia al reclamo histórico de las comunidades para poner en práctica sus derechos y también de satisfacer sus múltiples necesidades. El organismo gubernamental define su función como el reconocimiento de las demandas y de las necesidades a satisfacer a través de la gestión de los derechos de las comunidades establecidos por la Ley Nacional $\mathrm{N}^{\circ}$ 23.302/85 de creación del Instituto Nacional de Asuntos Indígenas. En este sentido, podemos interpretar que la legislación es reconocida como uno de los mecanismos para modificar las arquitecturas de gobierno excluyentes, e input posibilitador del ejercicio de los derechos de manera participativa en el marco del desarrollo con inclusión de las comunidades. 
Al concentrarnos en el NBI, encontramos que sus componentes nos permiten recuperar y discriminar ciertas problemáticas del área de producción rural (edilicias, sanitarias, la cantidad de miembros en el hogar, tenencia de la tierra, acceso al agua).

Los indicadores e índices censales señalan la vulnerabilidad estructural de la población, mientras que el análisis cualitativo permite acceder a las características que adquiere la vulnerabilidad de las comunidades situadas territorialmente.

La provincia de Río Negro tenía en el año 2010, 633.645 habitantes, con una densidad de 3,44 hab/ $\mathrm{km}^{2}$ (datos del Censo Nacional de Población, hogares y vivienda, INDEC 2010). El departamento con mayor densidad de población es General Roca (21.9 hab $/ \mathrm{km}^{2}$ ) y dentro de la Línea Sur, el área de estudio presenta valores muy bajos, los departamentos de Norquinco y 25 de Mayo, presentan una densidad de 0.2 y 0.6 hab $/ \mathrm{km}^{2}$ respectivamente (Lípori y De la Cal, 2014). En términos relativos, un valor alto en algún indicador socioeconómico o del NBI, al ponerlo en relación con la densidad poblacional muy baja, puede indicar que está afectando a toda la población. Mientras que si la densidad es alta, puede que ese indicador esté afectando a un grupo o zona determinada y concentrada en algún área del territorio, y no a toda la población.

Indagando entre los componentes del NBI, hemos observado mayor adecuación de los indicadores relacionados con algunas de las políticas públicas de intervención en el área de estudio. La tipología efectuada sobre un total de 81 (ochenta y uno) proyectos de la institución gubernamental, al correlacionarse con los componentes del NBI que se relacionan con los resultados de las entrevistas, observamos que los mismos dan cuenta de la reducción de algunos de los valores elevados de vulnerabilidad antes mencionados.

A partir del trabajo etnográfico, el trabajo de campo de gestión e incluso a partir de relevamientos de prensa, el problema más destacado por los productores rurales es la dificultad de acceso y extracción de agua. Este recurso aparece referido de manera directa e indirecta a través de referencias a otros elementos naturales como mallín, vertiente, precipitación, a prácticas afectadas por su falta como perforación, ganado, y de un fenómeno socioambiental como la sequía. Esta referencia en la relación comunidadgobierno, se traduce en los talleres de gestión, en demandas concretas que se derivan en mecanismos para acceder a planes y proyectos públicos. Encontramos que un $39 \%$ de los proyectos responde directa o indirectamente a cuestiones de vivienda y agua. 
De manera correlativa, la tipología de proyectos, expresa una respuesta a la demanda social más extendida a causa de la amenaza de la sequía amplificada por la caída de cenizas. Además, la variación en el número absoluto de proyectos relacionados con el acceso y extracción de agua indica que existió un incremento de estos para los años 2008 y 2011, lo que representa un $30 \%$ de los proyectos totales del período 2005 - 2014.

No obstante la rápida respuesta para aprobar los proyectos, la demora para su implementación es una referencia de la lentitud administrativa. Esto podría considerarse un indicador de las dificultades que tiene la gestión institucional, que se potencian ante la emergencia, especialmente en poblaciones vulnerables, con bajas posibilidades socioeconómicas de recuperarse de las pérdidas.

Es entonces que nos encontramos con una doble situación, mientras por un lado las instituciones gubernamentales tienen un intrincado y extenso circuito administrativo que retrasa la salida de los proyectos, por otro lado hay un incremento en la cantidad de los mismos ante la emergencia, visibilizando una problemática social del clima árido: la falta de agua e inclemencia del clima.

Entre 2005 y 2014, los 81 proyectos implementados por el Instituto Nacional de Asuntos Indígenas para las provincias de Río Negro y Neuquén tuvieron plazos variables de ejecución de 6 (seis) meses a 2 (dos) años. Ellos representan una inversión pública de alrededor de 34 millones de pesos. ${ }^{10}$

Su distribución temporal anual indica que los años 2009 (18 proyectos), 2005 (16 proyectos) y 2007 (11 proyectos) fueron los que concentraron la mayor demanda, y los años 2008 (18 proyectos), 2007 (16 proyectos) y 2005 (14 proyectos) los de la mayor puesta en ejecución. A su vez, entre 2008 y 2014 se ejecutaron nueve proyectos de más de \$ 1 millón cada uno (sumando entre ellos un poco más de \$ 18 millones, el 53,4\% del monto total aplicado entre 2005 y 2014 en los 81 proyectos promovidos por el INAI), de los cuales cuatro atendieron la provisión de agua, sumando un total de $\$ 7.156 .000$, y uno a la asistencia directa a las comunidades afectadas por la erupción del volcán Puyehue en 2011 por un poco más de 3 millones de pesos. Así, podemos decir que existe una

\footnotetext{
${ }^{10}$ El criterio aplicado como variable de normalización de los montos del financiamiento fue la variación del Índice de Precios al Consumidor (IPC) del INDEC, tomando como base de la serie el año $2014=100$, por considerarlo más preciso respecto a los precios internos de nuestro país.
} 
correlación entre esta concentración de acciones de la gestión pública con fenómenos extremos, como los efectos de la sequía y la caída de cenizas volcánicas de la erupción del cordón Caulle-Puyehue.

La problemática hídrica estructural de la región es el problema más constante, tanto en la "normalidad" como durante las catástrofes. En 2009 el INAI define con un grupo de comunidades un proyecto hídrico que atiende un problema constante en el marco del riesgo socioambiental del clima árido. El proyecto consistió en la implementación de diferentes tipos de extracción y encamisado de pozos de agua a lo largo de toda la Línea Sur de la provincia de Río Negro abarcando aproximadamente a unas 18 comunidades y cooperativas. Este proyecto fue llevado adelante por el Bloque de Organizaciones de la Línea Sur, la cual es un conjunto de cooperativas agrícolas de la zona, a la que se suman comunidades indígenas mapuches.

\section{Organización social y participación para una gestión sustentable}

Las estrategias socioculturales asociativas, constituyen indicadores que permiten reconocer de qué manera la organización social amplía el abanico de formas adaptativas para reducir la vulnerabilidad. Si bien el auto-reconocimiento de la identidad mapuche es relevante en la organización jurídico-administrativa de las comunidades, encontramos casos en los que, por consenso y aceptación, puede designarse como comunero a un criollo. La argumentación de los habitantes rurales es el reconocimiento de estar todos expuesto a los mismos embates socioambientales y económicos.

Aunque muchas veces se presentan conflictos entre aborígenes y criollos, casos como el de Don F. R. P., un criollo vecino de una de las comunidades, que da cuenta de mejores formas de integración. Él es, actualmente, un comunero aborigen, por adscripción personal y aceptación comunitaria. El evento, es una práctica en el espacio de comunalización, que permite incorporar la finca de un criollo a la comunidad mapuche, durante el relevamiento territorial. A partir de ello el criollo pudo acceder a herramientas legales para defender su continuidad en el territorio donde produce y habita en el marco de 
la ley 26.160/2006. Estas formas de organización participativa institucionalizan el ejercicio del derecho, y de la responsabilidad para su ejercicio de manera colectiva. ${ }^{11}$

La pertenencia a la comunidad contribuye a alcanzar la tenencia de la tierra, un derecho largamente reclamado. No obstante, como mencionamos anteriormente, la vulnerabilidad elevada se relaciona con la disponibilidad del acceso y extracción de agua para la producción. Esto último con la característica ecológica de la región, la aridez, expone a la población a las amenazas potenciales derivadas de eventos como las sequías y las cenizas volcánicas, que constituyen catástrofes. En este marco, la pertenencia a la comunidad también permite el acceso a los programas sociales para paliar las necesidades estructurales, especialmente cuando se ven exacerbadas ante situaciones catastróficas.

Los programas que se implementaron en la Línea Sur, y en particular en el área de estudio entre 2005 y 2014 tienen esta particularidad, resultaron de demandas de la comunidad, que fueron sistematizadas de acuerdo con el cumplimiento de una serie de requisitos que solicita el organismo de gobierno.

Como se puede apreciar, la documentación y la evaluación de la misma no cuenta con un diagnóstico integral que incluya la vulnerabilidad social estructural, las formas de adaptación locales, ni las características del medio, ni de los riesgos existentes en el área de donde provienen las demandas.

No obstante los proyectos aplicados son apreciados por los actores sociales involucrados, pues mejoran sus condiciones de vida en el corto plazo, ya sea a través de incorporar medidas de mitigación de los efectos de la sequía con obras de perforación o mejora de los pozos, o de adaptación tales como la transferencia de tecnología para extraer agua, proveerse de electricidad por paneles solares, medidas adaptativas centradas en el fortalecimiento asociativo como la organización cultural, la educación, el mejoramiento de los sistemas de comunicación, por ejemplo con la incorporación de radios de frecuencia BLU y FM u otros.

\footnotetext{
${ }^{11}$ La Ley Nacional 26.160/06 declara la “... emergencia en materia de posesión y propiedad... a las tierras que tradicionalmente ocupan las comunidades indígenas originarias del país, ...” y se suspenden "... la ejecución de sentencias, actos procesales o administrativos, cuyo objeto sea el desalojo o desocupación de las tierras...".
} 
La gestión desarrollada por la institución gubernamental, emplea la modalidad participativa centrada en el reconocimiento y ponderación de las necesidades para generar los consensos que las transforma en "demandas" que se tornan luego en intervenciones territoriales.

Los habitantes rurales, criollos y mapuches suelen enfrentar las mismas problemáticas, las respuestas desde el Instituto Nacional de Asuntos Indígenas, por razones de especificidad, están orientadas a las comunidades indígenas (predominantemente mapuches en esta zona). Pero además del sistema de comunidades, el estado estimula otras estrategias socioculturales asociativas como las cooperativas. De este modo, estas instituciones constituyen indicadores adaptativos para reducir la vulnerabilidad en espacios bajo riesgo socioambiental.

No obstante la participación comunitaria, la falta de diagnósticos integrados, que incluyan la problemática ambiental, es una debilidad en la gestión, por cuanto las mejores intenciones de ésta en el corto plazo, podrían tornarse en una mala adaptación a largo plazo, cuando la acumulación de problemas ambientales constituye un escenario de riesgos mayores, como la desertificación.

El sistema hídrico del área se alimenta de la nieve que ingresa en las napas subterráneas, no obstante no se conocen las características de dichas napas, que constituyen la fuente del recurso hídrico que permite la vida en la región. Ignorar o negar la incertidumbre del sistema socioambiental indica una limitante para cualquier política de desarrollo que se aplique en el área, pues las innovaciones adaptativas de corto plazo, rápidamente volverán a resultar insuficientes para la población y sus actividades productivas.

\section{Reflexiones finales}

Al revisar y seleccionar los indicadores de vulnerabilidad de manera conjunta comprobamos que algunos de los indicadores censales y derivados del trabajo etnográfico están en relación directa con los proyectos gubernamentales. Aunque los indicadores censales hacen referencia general a las características de la población, en particular el NBI lo hace respecto de carencias específicas. Los indicadores cualitativos resultantes del 
trabajo de campo - y aludidos en este artículo - ayudan a actualizar el significado de los resultados censales en relación con el medio socioambiental y a las catástrofes.

La correlación entre los indicadores de necesidades básicas insatisfechas censales y discursivos es significativa en torno del recurso hídrico. La periodicidad de esta falta de recurso indica la normalidad, que es exacerbada dentro de la catástrofe. Ante la aparición del Estado como un actor en el territorio, en las comunidades se resignifican los patrones de asociativos tradicionales.

Es así que la voluntad política del gobierno, se articula con las capacidades de las poblaciones para hacer frente a situaciones socioambientales de riesgo, como por ejemplo la organización en comunidades y cooperativas se articulan a la hora de aplicar medidas de respuesta en contextos de catástrofe.

El ejercicio de transdisciplinariedad desarrollado nos permite comprender y repensar las respuestas aplicadas en función del desarrollo de la población de la región árida. Es así que el resultado parcial obtenido en torno de la valorización de los proyectos, nos permite entender que se trata de partes de procesos sociales que podrían ser proyectados a corto, medio y largo plazo. También, la valorización por parte de los beneficiarios nos acerca a la comprensión de las limitantes que los condicionan para garantizar la reducción de la vulnerabilidad, el ejercicio de los derechos y para generar medidas y comportamientos adaptativos que reduzcan el impacto de eventos extremos.

Las medidas y proyectos de corto plazo que identificamos refieren principalmente a la atención de las necesidades inmediatas. En casos de emergencia, el Estado ofrece el proyecto a las comunidades y cooperativas, y éstas deciden la forma de implementación. Por ejemplo los proyectos productivos de autoconsumo para garantizar la seguridad alimentaria humana o la provisión de alimento para animales en el momento cercano o durante la caída de ceniza.

Mientras que los proyectos con resultados a mediano plazo, incorporan un valor adicional para acceder al control de los recursos, su desarrollo a través de procesos participativos y atribución de responsabilidades refuerzan sus capacidades y protagonismo en procesos de negociación, a partir de demandas consensuadas, tanto en los mercados como en las estructuras políticas. Estos procesos conducen, aunque de manera indirecta, a 
identificar las vulnerabilidades propias y ejercer el consenso como mecanismo. Entre los proyectos que fortalecen el empoderamiento y la posibilidad de planificar su propio desarrollo, se encuentran aquellos de provisión de animales para la venta y reproducción, mejoramiento predial, pozos de agua, paneles solares, instalación de bombas, movilidad y comunicaciones.

En tanto que los proyectos con efectos de largo plazo son aquellos que, además del empoderamiento, redundan en una reorganización social y en el estímulo al desarrollo productivo en la región, como por ejemplo la tenencia de la tierra, la adquisición de residencias urbanas y el acceso a los servicios como educación y salud.

La buena proyección de estas medidas, queda limitada si no se toman en cuenta tempranamente las prácticas cotidianas que forman parte de las causas del riesgo. La institución considera las demandas/aspiraciones de las comunidades, pero ello no basta para garantizar la sostenibilidad de los proyectos, cuando para el diseño y puesta en práctica se necesita de técnicos externos a la comunidad.

El ejercicio participativo contribuye por un lado al empoderamiento de la sociedad, y forma parte del objetivo de gobernanza e institucionalización que le permite ejercer el derecho a la organización comunitaria. El mismo proceso exigido para la asignación de recursos, contribuye significativamente a la percepción y al conocimiento de su propia vulnerabilidad.

Entre las limitantes institucionales, pese al intento de universalización de los derechos, consiste en que el ejercicio supone la carencia como hecho total, y en su intervención no discrimina a las comunidades por su vulnerabilidad estructural. Esto nos permite inferir que lo importante para el Estado es la personería jurídica, y las amenazas que constituyen la situación de riesgo socioambiental solo configuran coyunturas.

Para el caso del área de influencia del sitio Ingeniero Jacobacci, caracterizado por el estrés hídrico y mayoría de población mapuche rural, las políticas institucionales para acceso y extracción del agua están enmarcadas en el mismo proceso de riesgo como respuestas que tienden a la mitigación de los síntomas que dejó la situación catastrófica o extrema. 
A partir de la exploración conjunta, entendemos que la gestión del riesgo debe formar parte del diseño del desarrollo social, especialmente cuando se busca incrementar las capacidades de las poblaciones para mejorar sus condiciones de reproducción social y económica en la "normalidad". Entendemos que para optimizar el desarrollo social habría quereducir las incertidumbres a través de diagnosticar la vulnerabilidad social estructural y en sus formas de adaptación, explorando la correlación entre las prácticas productivas y sus efectos sobre los recursos vitales como el suelo y el agua durante la normalidad.

Estas consideraciones permitirían elaborar una prospectiva de la potencialidad de sus resultados en diferentes escenarios. Tanto reconocer la capacidad para mitigación de efectos no deseados pero inminentes, como de prevención para mejorar las condiciones adaptativas que tiendan, a mediano y largo plazo, a reducir los aspectos centrales de la vulnerabilidad, identificar y actuar tempranamente sobre las causas del riesgo, como la sequía y la caída de cenizas.

\section{Referencias}

ACI (Alianza Cooperativa Internacional). Declaración sobre Identidad y Principios Cooperativos, adoptados en Manchester en 1995.

BROW, James. (1990). Notes on Community, Hegemony, and Uses of the Past. Anthropological Quarterly, v. 63, p. 1-5, 1990.

DOUGLAS, Mary. La aceptabilidad del riesgo. Paidós. España, 1996.

GARCÍA ACOSTA, Virginia. Historical Disaster Research. En: S.M. Hoffman y A. Oliver-Smith (eds.). Catastrophe \& Culture. The Anthropology of Disaster. School of American Research/James Currey Ltd., Santa Fe/Oxford, p. 49-66, 2002.

GARCÍA ACOSTA, Virginia. El riesgo como construcción social y la construcción social de riesgos. Desacatos. Revista de Antropología Social. 19, septiembre-diciembre, p. 11-24, 2005.

GIBBONS, Michael; LIMOGES, Camille; NOWOTNY, Helga; SCHWARTZMANN, Simon; SCOTT, Peter and TROW, Martin. The New Production of Knowledge: the Dynamics of Science and Research in Contemporary Societies. London: SAGE, 1994.

INDEC (Instituto Nacional de Estadísticas y Censos- República Argentina). Censo Nacional de Población y Viviendas 2010. http://www.censo2010.indec.gov.ar.

LADA. Evaluación de la Desertificación en Argentina. Resultados del Proyecto LADA/FAO. Buenos Aires: Gráfica Latina S.A., 2011. Disponible en http://www.desertificacion.gob.ar/documentacion/publicacion/nivel-nacional/page/2/, Consultado en junio de 2013.

LAVELL, Allan. Local Level Risk Management: From Concept to Practice. Quito: CEPREDENAC-UNDP, 2005.

LAVELL, Allan. Relationships between Local and Community Disaster Risk Management \& Poverty Reduction: A Preliminary Exploration. A Contribution to the 2009 ISDR Global Assessment Report on Disaster Risk Reduction. 2009. 
LÍPORI, Mariana. Caracterización de la vulnerabilidad en contexto de amenaza. Mesa Cambio global: riesgos y desafíos en los nuevos escenarios socioambientales. XI Congreso Argentino de Antropología Social. Rosario, 23 al 26 de Julio de 2014.

MURGIDA, Ana; GONZALEZ, Marcela y TIESSEN, Holm. Rainfall trends, land use and adaptation in the Chaco salteño region of Argentina. Journal Climate Change, v. 14, n. 4, p. 13871394, 2014.

MURGIDA, Ana y GENTILE, Elvira. Aceptabilidad y amplificación del riesgo en la estepa norpatagónica. En Viand, J. Y Briones, F. Riesgos al Sur. Diversidad de riesgos de desastres en Argentina. Buenos Aires: Red de Estudios Sociales en Prevención de Desastres en América Latina/ Imago Mundi Editores, 2015.

RIBEIRO, Darcy. El proceso civilizatorio: de la revolución agrícola a la termonuclear. Buenos Aires: Centro Editor de América Latina, 1971.

ROUX, Dirk; ROGERS, Kevin; BIGGS, Harry; ASHTON, Peter and SERGEANT, Anne. Bridging the science-management divide: Moving from unidirectional knowledge transfer to knowledge interfacing and sharing, Ecology and Society, v. 11, n. 1, 2006. [online] URL: http://www.ecologyandsociety.org/vol11/iss1/art4/

SCHMALE, Julia, MAAS, Achim, CHABAY, Ilan, LAWRENCE, Mark G. Co-designing Usable Knowledge with Stakeholders and Fostering Ownership - A Pathway through the communication problem? En: Impacts World 2013 Conference Proceedings. Potsdam: Potsdam Institute for Climate Impact Research, p. 852-860, 2013.

TÁBARA, Joan David and CHABAY, Ilan. Coupling Human Information and Knowledge Systems with social-ecological systems change: Reframing research, education, and policy for sustainability, Environmental Science and Policy, v. 20, p. 71-81, 2013.

UNEP 2004. Freshwater in Europe. Facts, figures and maps. 2004, United Nations Environment Programme - DEWA Europe. http://www.grid.unep.ch/products/3_Reports/freshwater_atlas.pdf.

\section{Legislación citada}

Ley Nacional $\mathrm{N}^{\circ} 26.160$ sancionada en el 2006.

Ley 23.302/85 de creación del INAI.

Resolución $N^{\circ} 781 / 1995$ de la Secretaría de Desarrollo Social de la Nación.

Recebido em: 10/11/2015.

Aprovado em: 07/03/2016.

Iluminuras, Porto Alegre, v. 17, n. 41, p.11-29, jan/jun, 2016. 